\title{
Review Article \\ Effect of Anti-TNF Antibodies on Clinical Response in Rheumatoid Arthritis Patients: A Meta-Analysis
}

\author{
Chunxiao Wu, ${ }^{1}$ Shengxu Wang, ${ }^{1}$ Peifeng Xian, ${ }^{1}$ Lu Yang, ${ }^{1,2}$ Ying Chen, ${ }^{1,2}$ and Xianjie Mo ${ }^{2}$ \\ ${ }^{1}$ School of Traditional Chinese Medicine, Southern Medical University, Guangzhou, Guangdong 510515, China \\ ${ }^{2}$ Traditional Chinese Medicine-Integrated Hospital, The Affiliated Hospital of Southern Medical University, \\ Guangzhou, Guangdong 510315, China
}

Correspondence should be addressed to Lu Yang; yl800526@163.com and Ying Chen; yingchenacut@163.com

Received 11 April 2016; Accepted 1 June 2016

Academic Editor: Toshihiro Nanki

Copyright (C) 2016 Chunxiao Wu et al. This is an open access article distributed under the Creative Commons Attribution License, which permits unrestricted use, distribution, and reproduction in any medium, provided the original work is properly cited.

\begin{abstract}
Background. Antitumor necrosis factor (anti-TNF) drugs have been applied for rheumatoid arthritis (RA) treatment; however, patients having anti-drug antibodies (ADAbs) do not benefit from these drugs. The meta-analysis aims to comprehensively assess the relationship between $\mathrm{ADAb}$ positive $(\mathrm{ADAb}+)$ and anti-TNF response in RA patients. Methods. Observational studies comparing different clinical response between $\mathrm{ADAb}+$ and $\mathrm{ADAb}$ negative groups were included. Odds ratio (OR) with its corresponding 95\% confidence interval (CI) was used as effect size. Subgroup analyses stratified by TNF inhibitor types and assay methods for ADAb detection were performed. Results. Totally, 10 eligible studies containing 1806 subjects were included. ADAb+ was significantly associated with reduced anti-TNF response to RA at all the time points after follow-up $(P<0.001)$. Subgroup analysis also supported this significant association $(P<0.05)$, except for enzyme-linked immunosorbent assay (ELISA) group at 3 months, infliximab (INF) and enzyme-linked immunosorbent assay (ELISA) groups at 6 months, and Immunological MultiParameter Chip Technology (IMPACT) group at 12 months. Conclusion. ADAb+ was significantly associated with reduced clinical response in RA patients, and other alternatives should be considered in RA patients presenting ADAb+.
\end{abstract}

\section{Introduction}

Rheumatoid arthritis (RA) is one of the autoimmune and chronic diseases [1]. Characterized by inflammation and destruction of joints, RA has caused increased mortality and substantial economic burden on patients worldwide [2].

Biologic therapies that target a specific inflammatory pathway or immune-related system could improve outcomes of RA patients, contributing to reduced mortality and comorbidity [3]. The tumor necrosis factor (TNF) encoded protein is a proinflammatory cytokine that is generated by macrophages in response to endotoxin [4]. Anti-TNF agents were firstly confirmed as the biologic drugs for RA treatment when nonbiologic disease modified antirheumatic drugs (DMARDs) were failing [5]. At present, five anti-TNF drugs have been licensed for the clinical use of RA, including infliximab (INF), adalimumab (ADAL), etanercept (ETN), golimumab (GLM), and certolizumab (CTZ) [6-9]. However, since TNF has significant roles in host defense, numerous studies find that anti-TNF application is also related to increased risk of serious infections and malignancies in RA patients [10-12]. Moreover, several patients who generate the anti-drug antibodies (ADAbs) do not benefit from the antiTNF drugs [13].

Previously, several meta-analyses have been carried out to evaluate the safety of anti-TNF agents. For instance, Bongartz et al. discovered that two anti-TNF agents, INF and ADAL, could result in increased risk of malignancies in RA patients in a dose-dependent manner [6]. Another meta-analysis indicates anti-TNF- $\alpha$ is correlated with decreased risk of all cardiovascular events [14]. Aaltonen et al. compare the efficacy and safety of anti-TNF drugs with methotrexate and find that there are no significant differences between them in efficacy, while ETN has a reduced risk of adverse event and is proposed as the safest alternative [15]. However, causative factors for these results are not considered, nor the drug immunogenicity or ADAb. In addition, different time points in these studies might produce different results. 


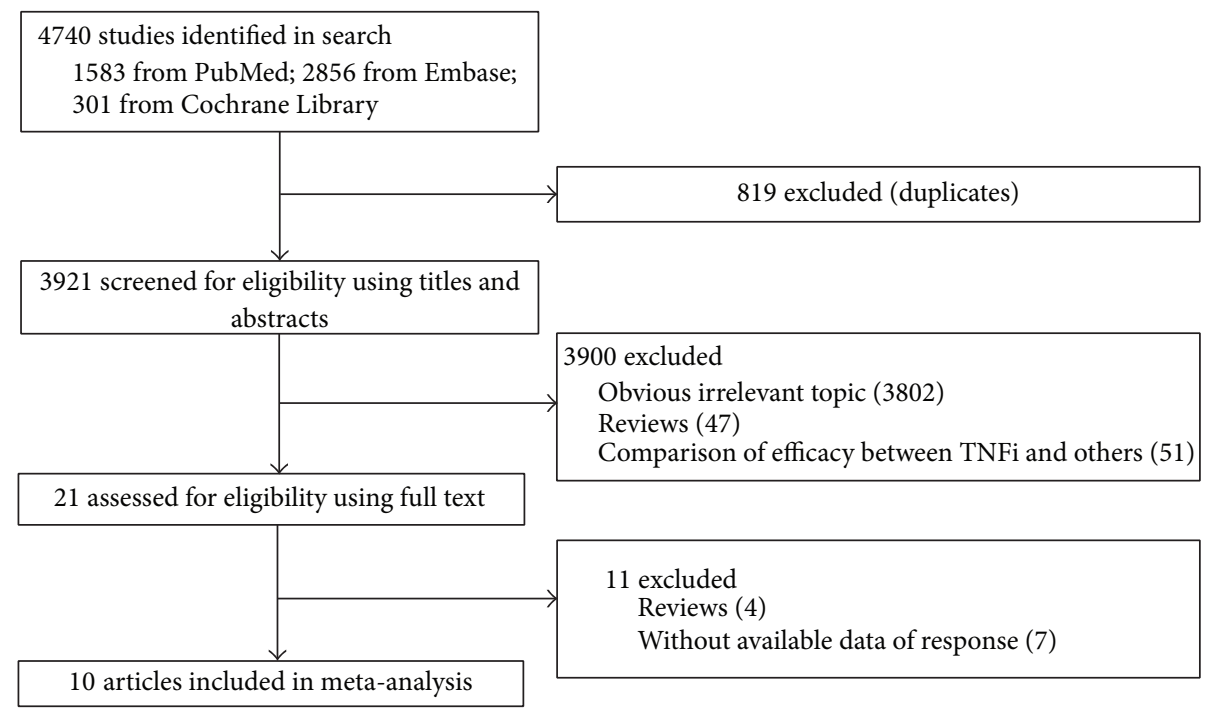

FIgURE 1: Procedures of study selection.

Therefore, we performed this meta-analysis, mainly concerning the ADAb status (ADAb positive or negative), to comprehensively assess association between $\mathrm{ADAb}+$ and response to anti-TFN agents, aiming to give a precise assessment of application of anti-TNF for RA management.

\section{Methods}

2.1. Search Strategy. Literature search was conducted in databases such as PubMed, Embase, and Cochrane Library. The search strategy was ("Immunogenicity" OR "response") AND ("rheumatoid arthritis" OR "RA") AND ("anti-tumor necrosis factor" OR “anti-TNF” OR “TNF- $\alpha$ antagonist” OR “TNF inhibitors" OR "infliximab" OR "adalimumab” OR “etanercept" OR “golimumab” OR “certolizumab” OR “infliximab biosimilar") AND ("antibody" OR "ADAb"). There was no language restriction and the searching was set before January 5, 2016. Additionally, manual search for studies that were published in paper was conducted. Reference lists of included studies were also scanned for more eligible studies.

2.2. Inclusion and Exclusion Criteria. The inclusion criteria were as follows: (1) subjects were RA patients $\geq 18$ years old; (2) chemotherapies were anti-TNF agents such as INF, ADAL, ETN, GLM, and CTZ; (3) the studies compared therapeutic differences between $\mathrm{ADAb}$ positive $(\mathrm{ADAb}+)$ and $\mathrm{ADAb}$ negative (ADAb-) RA patients; (4) the outcome was clinical response with the measurement criteria of the American College of Rheumatology (ACR) or European League Against Rheumatism (EULAR) criteria for RA; (5) the study type was observational study.

The exclusion criteria were as follows: (1) no control group was contained in the study; (2) data were incomplete or the results could not be used for statistical analysis; (3) the studies were reviews, letters, or comments.
2.3. Data Extraction. Two investigators independently completed the literature selection based on the predefined criteria. Then, the following required data were abstracted, such as first-author name, publication year, study region, TNF types, subjects' characteristics (e.g., sample size, age, gender composition, and course of disease), time point in observational studies, sample sizes, and case numbers of outcomes in $\mathrm{ADAb}+$ group and $\mathrm{ADAb}$ - group, respectively. Disagreements were resolved through discussion with a third investigator.

2.4. Statistical Analysis. Odds ratio (OR) with its corresponding $95 \%$ confidence interval (CI) was used as a measure of the effect size to calculate differences of clinical response between $\mathrm{ADAb}+$ and $\mathrm{ADAb}-$ groups. Heterogeneities across studies were determined by Cochrane's $Q$ statistic and $I^{2}$ test [16]. If extensive heterogeneity was detected $\left(P<0.05, I^{2}>50 \%\right)$, a randomized-effects model was applied, whereas if there was no obvious heterogeneity $\left(P>0.05, I^{2} \leq 50 \%\right)$, a fixed-effects model was applied.

Outcomes were pooled at different time points ( 3 months, 6 months, 12 months, and $\geq 24$ months) after follow-up, respectively. Subgroup analyses stratified by TNF inhibitor (TNFi) type and assay method for ADAb detection were performed to further explore influences of these specific factors on the outcomes. Publication bias was determined using Egger's test [17]. The Statall.0 software (STATA, College Station, TX, USA) was used to complete all the statistical analyses.

\section{Results}

3.1. Eligible Studies. Specific procedures of the study selection are listed in Figure 1. By the preliminary search, a total of 4740 studies were retrieved, and 3921 remained after eliminating 
duplicated publications. Through title and abstract screening, another 3900 studies (including 3802 studies that obviously did not conform to the inclusion criteria, 47 reviews, and 51 studies that compared the therapeutic effects of TNFi with other drugs on RA) were excluded. Eleven out of the remaining 21 studies were removed after full-text reading. No additional studies were added via manual search. As a result, 10 eligible studies [18-27] were included in the meta-analysis.

3.2. Characteristics of the Included Studies. As indicated in Table 1, the 10 studies consisted of a total of 1806 subjects. These studies were published from 2007 to 2015, and most of them were conducted in European countries except Chen et al's study [20], which was carried out in China. Three ADAb detection methods, radioimmunoassay (RIA), enzymelinked immunosorbent assay (ELISA), and Immunological Multi-Parameter Chip Technology (IMPACT), and 6 time points of the outcomes, including 3, 6, 12, 24, 36, and 48 months after follow-up, were involved.

3.3. Meta-Analysis and Subgroup Analysis. A fixed-effects model was used for comparisons at the time points of 3 , $12,24,36$, and 48 months after follow-up, due to the lack of significant heterogeneity $\left(P>0.05, I^{2} \leq 50 \%\right)$. By contrast, a randomized-effects model was applied 6 months after follow-up due to significant heterogeneity $\left(I^{2}=72.7 \%\right.$, $P=0.005)$. As expected, ADAb+ was significantly associated with reduced anti-TNF response to RA at all the time points after follow-up ( 3 months: $\mathrm{OR}=0.03,95 \% \mathrm{CI}$ : 0.01 to 0.13 , $P<0.001 ; 6$ months: $\mathrm{OR}=0.04,95 \% \mathrm{CI}: 0.01$ to $0.22, P<$ $0.001 ; 12$ months: $\mathrm{OR}=0.26,95 \% \mathrm{CI}$ : 0.11 to $0.57, P<0.001$; $\geq 24$ months: $\mathrm{OR}=0.16,95 \% \mathrm{CI}: 0.08$ to $0.33, P<0.001$ ) (Figure 2).

Subgroup analysis stratified by TNFi types and assay methods also supported this significant association $(P<$ 0.05 ), except for ELISA group at 3 months (OR $=0.10,95 \%$ CI, 0.01 to 2.41$)$, INF (OR $=0.05,95 \% \mathrm{CI}, 0.00$ to 1.06$)$ and ELISA (OR $=0.05,95 \% \mathrm{CI}, 0.00$ to 1.13 ) groups at 6 months, and IMPACT group at 12 months $(\mathrm{OR}=0.66,95 \% \mathrm{CI}, 0.35$ to 1.24) (Table 2).

3.4. Publication Bias. As only a few studies compared the clinical response between the two groups at 3,6 , and $\geq 24$ months after follow-up, we just evaluated the publication bias at the time point of 12 months. Egger's test indicated that there lacked significant publication bias $(P=0.067)$.

\section{Discussion}

In the present study, a total of 10 studies were included involving 1806 subjects. Meta-analysis indicated that ADAb+ group had significant association with reduced anti-TNF response $(P<0.05)$, whatever the time point was. Moreover, in most of the subgroups stratified by TNFi types and assay methods, significant association was also detected between $\mathrm{ADAb}+$ group and decreased anti-TNF response, except in ELISA group at 3 months, INF and ELISA groups at 6 months, and IMPACT group at 12 months. Studies that used RIA assay all confirmed this significant association at each time point.
Five anti-TNF drugs have been approved for RA treatment. A large number of randomized clinical trials and observational studies have confirmed the effectiveness of anti-TNF agents for RA treatment, whether at an early stage or during a long period [28-30]. However, multiple studies point out that the RA patient prescribed these drugs is at an increased risk of serious infections and malignancies [3133]. In addition, in several patients, anti-TNF agents are ineffective for RA management [34]. ADAb generated in patients is one causative factor for nonresponse to anti-TNF drugs such as INF and ADAL for RA treatment $[35,36]$. Reportedly, more INF doses are needed in RA patients with anti-INF antibodies, and high ADAb level of anti-INF is related to the loss of clinical response, which would discount the therapeutic efficacy [25]. Several studies indicate that different bioavailability and diverse development of ADAb in RA patients may be the major reasons for different clinical responses [37, 38].

Immune response to anti-TNF therapy contributes to the generation of ADAbs, which could impair the clinical response if they are able to decrease the serum levels of the active drug [39]. Immunogenicity of the anti-TNF agents is considered as the main mechanism of treatment failure [40]. The immunogenicity rate of different anti-TNF drugs is different, due to diversities in various factors such as administration route, drug dose, concomitant medication, detailed treatment schedule, and immune and nutritional status [41]. Extensive studies have investigated influence of the immunogenicity of anti-TNF agents on drug efficacy and safety and found that antibodies of these agents, such as INF, could affect the drug's pharmacokinetics, which may lead to delayed infusion and injection site reactions [41, 42]. Meanwhile, the anti-chimeric antibodies (ATIs), induced by INF, could increase the clearance of INF and attenuate its function [43]. This might be the reasonable explanation for our finding of the significant association between $\mathrm{ADAb}+$ and reduced response rate.

On the other hand, the detection of ADAb might be influenced by different collection times and methods. For instance, ATIs may be undetectable at the initiation of INF administration due to the fact that they generate the immune complexes with the drug [41]. With regard to the detection methods, a bridging ELISA is the most common assay for $\mathrm{ADAb}$ detection [34]; however, it could be influenced by a high rate of false positive results [44]. A study indicates that an underestimated $\mathrm{ADAb}+$ rate might be due to undetectable $\mathrm{ADAb}$ using the bridging ELISA [45]. Therefore, it is understandable that, in ELISA group at 3 months and 6 months, there was not any observed association between ADAb+ and reduced response, which may be attributed to the reduced detection rate by ELISA method.

IMPACT is a multiplex platform that is a novel technology for $\mathrm{ADAb}$ detection, and, reportedly, it has greater sensitivity for ADAb detection, compared with other current clinical tests [46]. However, the duration in this study is as short as $<6$ months. Another study points out that IMPACT is a novel method mainly used for definition of clinical stages of RA at molecular level [47]. Therefore, whether this method 


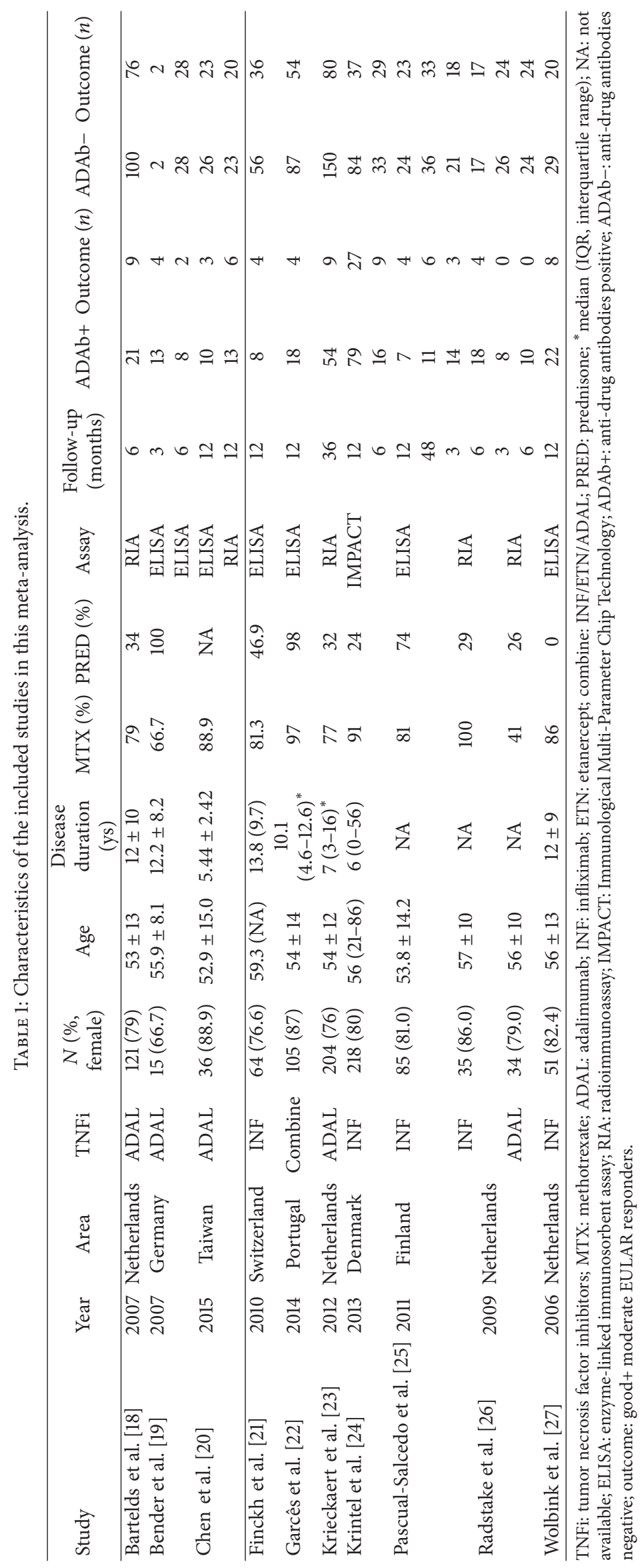




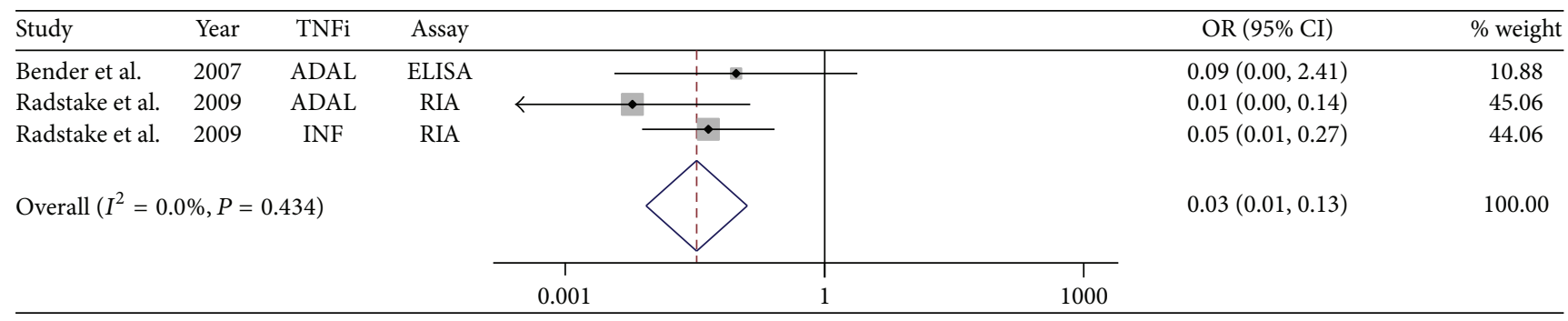

(a)

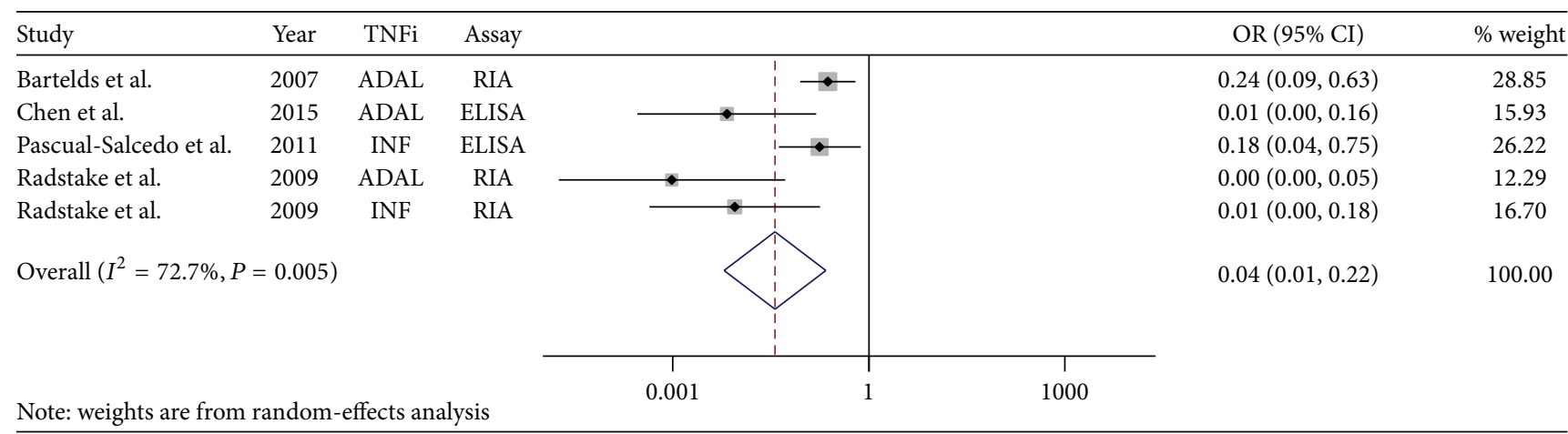

(b)

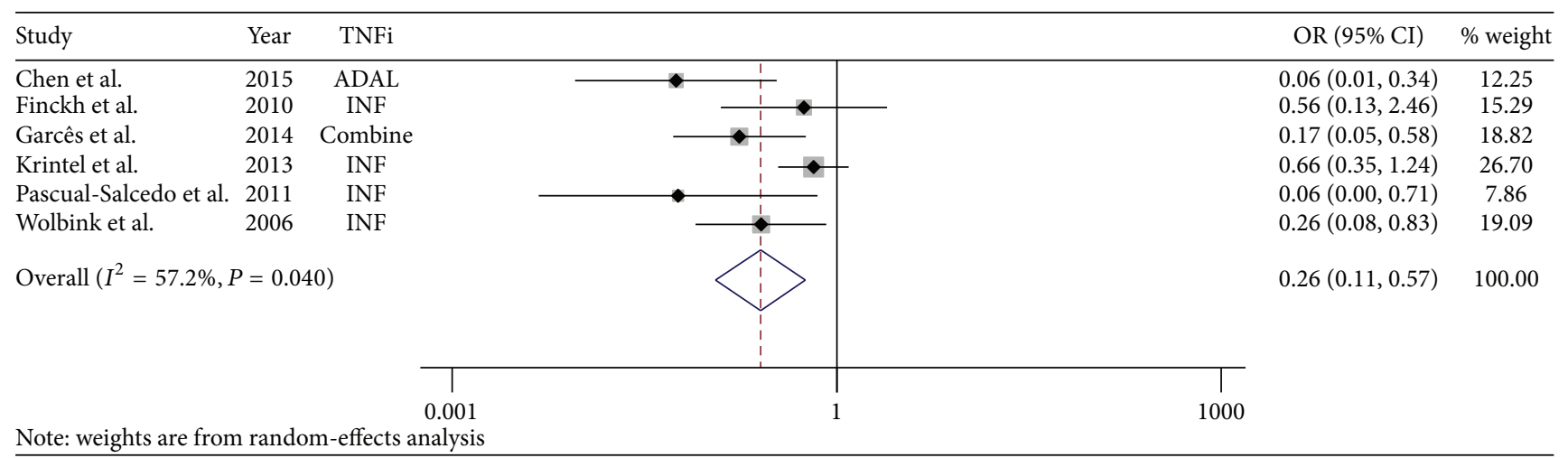

(c)

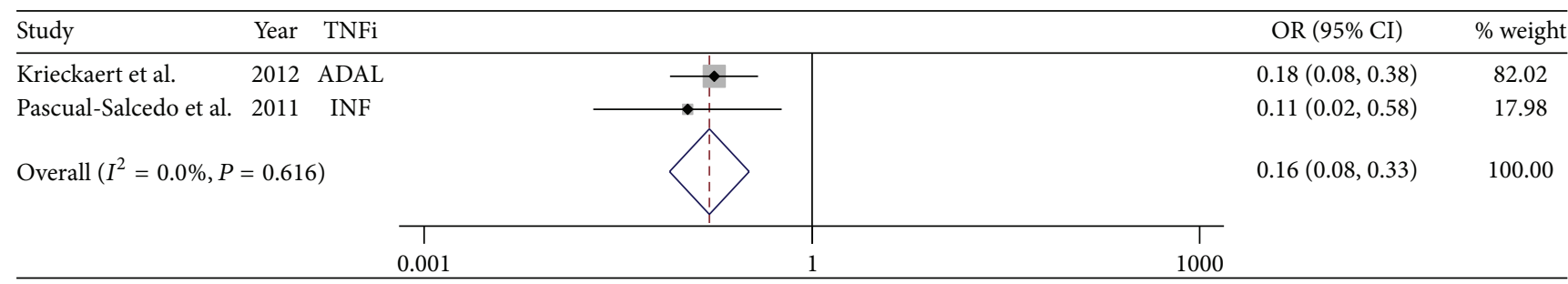

(d)

FIGURE 2: Effect of ADAb+ on anti-TNF response in rheumatoid arthritis patients, compared with ADAb- group at different time points after follow-up. (a) At 3 months; (b) at 6 months; (c) at 12 months; (d) at $>24$ months.

is precise and sensitive in a long duration needs to be further investigated. Our discovery that, in IMPACT group at 12 months, $\mathrm{ADAb}+$ was not associated with decreased clinical response provides a hint that IMPACT might not be sensitive for ADAb detection at a long duration. However, it needs to be verified by more studies. For RIA assay, a previous study that assessed INF levels and ADAbs for a long duration (1.5 to 18 months) using different methods found that fluid-phase RIA was better than cross-binding ELISA [48]. Therefore, it is understandable that whatever the time point, $\mathrm{ADAb}$ was significantly associated with reduced anti-TNFi clinical response using this detection method. 


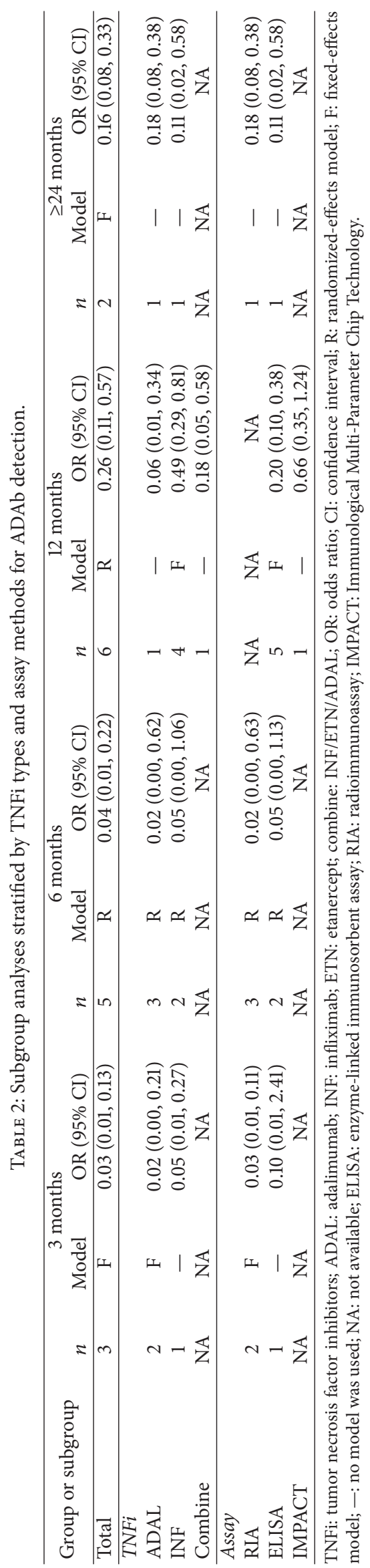


Our study has the following advantages: (1) the clinical response was pooled at different time points, respectively, which avoided potential bias generated from drug effect varied by different time points; (2) subgroup analyses stratified by TNFi types and assay methods for ADAb detection were performed, contributing to exploration of more precise correlations between $\mathrm{ADAb}+$ and response to specific antiTNF drugs in RA patients; (3) no obvious publication bias was observed in this meta-analysis, indicating the reliability of our results. However, there were also several limitations as follows: (1) all the included studies were observational studies, and the confounding factors could not be well controlled; (2) significant heterogeneity was detected at several time points, which might cause deviation to some extent; (3) drug level in different studies might be a confounding factor that influences the result; however, it was not taken into consideration in our study; (4) the sample size was relatively small, and large-scale studies with more samples are required to confirm our findings.

In conclusion, $\mathrm{ADAb}+$ was significantly associated with reduced clinical response in RA patients, and other alternatives should be considered in RA patients presenting ADAb+.

$\begin{array}{ll}\text { Abbreviations } \\ \text { TNF: } & \text { Tumor necrosis factor } \\ \text { ADAb+: } & \text { Anti-drug antibody positive } \\ \text { ADAb-: } & \text { Anti-drug antibody negative } \\ \text { OR: } & \text { Odds ratio } \\ \text { CI: } & \text { Confidence interval } \\ \text { TNFi: } & \text { TNF inhibitor } \\ \text { INF: } & \text { Infliximab } \\ \text { ADAL: } & \text { Adalimumab } \\ \text { ETN: } & \text { Etanercept } \\ \text { Combine: } & \text { INF/ETN/ADAL } \\ \text { ELISA: } & \text { Enzyme-linked immunosorbent assay } \\ \text { RIA: } & \text { Radioimmunoassay } \\ \text { IMPACT: } & \text { Immunological Multi-Parameter Chip } \\ & \text { Technology. }\end{array}$

\section{Disclosure}

Chunxiao $\mathrm{Wu}$ is the first author and Shengxu Wang is co-first author. Lu Yang is the corresponding author and Ying Chen is the co-corresponding author.

\section{Competing Interests}

The authors declare that there are no competing interests regarding the publication of this paper.

\section{Authors' Contributions}

Lu Yang obtained funding. Lu Yang and Ying Chen participated in the study concept and design and paper authorization. Peifeng Xian, Chunxiao Wu, Xianjie Mo, and Ying Chen assessed studies. Lu Yang and Shengxu Wang analyzed the data. Chunxiao Wu and Shengxu Wang wrote the paper and ensured the integrity of the data.

\section{Acknowledgments}

This study was supported by the National Natural Science Foundation of China (no. 81303047).

\section{References}

[1] M. Feldmann, "Development of anti-TNF therapy for rheumatoid arthritis," Nature Reviews Immunology, vol. 2, no. 5, pp. 364-371, 2002.

[2] R. Fleischmann, J. Kremer, J. Cush et al., "Placebo-controlled trial of tofacitinib monotherapy in rheumatoid arthritis," New England Journal of Medicine, vol. 367, no. 6, pp. 495-507, 2012.

[3] B. B. Aggarwal, "Signalling pathways of the TNF superfamily: a double-edged sword," Nature Reviews Immunology, vol. 3, no. 9, pp. 745-756, 2003.

[4] K. Chaabo and B. Kirkham, "Rheumatoid arthritis-anti-TNF," International Immunopharmacology, vol. 27, no. 2, pp. 180-184, 2015.

[5] J. B. Galloway, K. L. Hyrich, L. K. Mercer et al., "Anti-TNF therapy is associated with an increased risk of serious infections in patients with rheumatoid arthritis especially in the first 6 months of treatment: updated results from the British Society for Rheumatology Biologics Register with special emphasis on risks in the elderly," Rheumatology, vol. 50, no. 1, pp. 124-131, 2011.

[6] T. Bongartz, A. J. Sutton, M. J. Sweeting, I. Buchan, E. L. Matteson, and V. Montori, "Anti-TNF antibody therapy in rheumatoid arthritis and the risk of serious infections and malignancies: systematic review and meta-analysis of rare harmful effects in randomized controlled trials," The Journal of the American Medical Association, vol. 295, no. 19, pp. 22752285,2006

[7] D. J. Lovell, A. Reiff, N. T. Ilowite et al., "Safety and efficacy of up to eight years of continuous etanercept therapy in patients with juvenile rheumatoid arthritis," Arthritis and Rheumatism, vol. 58, no. 5, pp. 1496-1504, 2008.

[8] E. C. Keystone, M. C. Genovese, L. Klareskog et al., "Golimumab, a human antibody to tumour necrosis factor $\alpha$ given by monthly subcutaneous injections, in active rheumatoid arthritis despite methotrexate therapy: the GO-FORWARD Study," Annals of the Rheumatic Diseases, vol. 68, no. 6, pp. 789796, 2009.

[9] R. Fleischmann, J. Vencovsky, R. F. Van Vollenhoven et al., "Efficacy and safety of certolizumab pegol monotherapy every 4 weeks in patients with rheumatoid arthritis failing previous disease-modifying antirheumatic therapy: the FAST4WARD study," Annals of the Rheumatic Diseases, vol. 68, no. 6, pp. 805811, 2009.

[10] S. A. A. Van Dartel, J. Fransen, W. Kievit et al., "Predictors for the 5-year risk of serious infections in patients with rheumatoid arthritis treated with anti-tumour necrosis factor therapy: a cohort study in the Dutch Rheumatoid Arthritis Monitoring (DREAM) registry," Rheumatology, vol. 52, no. 6, pp. 1052-1057, 2013.

[11] J. R. Curtis, F. Xie, L. Chen et al., "Use of a disease risk score to compare serious infections associated with anti-tumor necrosis factor therapy among high-versus lower-risk rheumatoid 
arthritis patients," Arthritis Care and Research, vol. 64, no. 10, pp. 1480-1489, 2012.

[12] J. P. Leombruno, T. R. Einarson, and E. C. Keystone, "The safety of anti-tumour necrosis factor treatments in rheumatoid arthritis: meta and exposure-adjusted pooled analyses of serious adverse events," Annals of the Rheumatic Diseases, vol. 68, no. 7, pp. 1136-1145, 2009.

[13] M. E. Weinblatt, E. C. Keystone, D. E. Furst et al., "Adalimumab, a fully human anti-tumor necrosis factor $\alpha$ monoclonal antibody, for the treatment of rheumatoid arthritis in patients taking concomitant methotrexate: the ARMADA trial," Arthritis \& Rheumatism, vol. 48, no. 1, pp. 35-45, 2003.

[14] C. Barnabe, B.-J. Martin, and W. A. Ghali, "Systematic review and meta-analysis: anti-tumor necrosis factor $\alpha$ therapy and cardiovascular events in rheumatoid arthritis," Arthritis Care \& Research, vol. 63, no. 4, pp. 522-529, 2011.

[15] K. J. Aaltonen, L. M. Virkki, A. Malmivaara, Y. T. Konttinen, D. C. Nordström, and M. Blom, "Systematic review and metaanalysis of the efficacy and safety of existing TNF blocking agents in treatment of rheumatoid arthritis," PLOS ONE, vol. 7, no. 1, Article ID e30275, 2012.

[16] J. P. T. Higgins, S. G. Thompson, J. J. Deeks, and D. G. Altman, "Measuring inconsistency in meta-analyses," British Medical Journal, vol. 327, no. 7414, pp. 557-560, 2003.

[17] M. Egger, G. D. Smith, and A. N. Phillips, "Meta-analysis: principles and procedures," The British Medical Journal, vol. 315, no. 7121, pp. 1533-1537, 1997.

[18] G. M. Bartelds, C. A. Wijbrandts, M. T. Nurmohamed et al., "Clinical response to adalimumab: relationship to antiadalimumab antibodies and serum adalimumab concentrations in rheumatoid arthritis," Annals of the Rheumatic Diseases, vol. 66, no. 7, pp. 921-926, 2007.

[19] N. K. Bender, C. E. Heilig, B. Dröll, J. Wohlgemuth, F.P. Armbruster, and B. Heilig, "Immunogenicity, efficacy and adverse events of adalimumab in RA patients," Rheumatology International, vol. 27, no. 3, pp. 269-274, 2007.

[20] D.-Y. Chen, Y.-M. Chen, W.-C. Tsai et al., "Significant associations of antidrug antibody levels with serum drug trough levels and therapeutic response of adalimumab and etanercept treatment in rheumatoid arthritis," Annals of the Rheumatic Diseases, vol. 74, no. 3, article e16, 2015.

[21] A. Finckh, J. Dudler, F. Wermelinger et al., "Influence of antiinfliximab antibodies and residual infliximab concentrations on the occurrence of acquired drug resistance to infliximab in rheumatoid arthritis patients," Joint Bone Spine, vol. 77, no. 4, pp. 313-318, 2010.

[22] S. Garcês, M. Antunes, E. Benito-Garcia, J. C. da Silva, L. Aarden, and J. Demengeot, "A preliminary algorithm introducing immunogenicity assessment in the management of patients with RA receiving tumour necrosis factor inhibitor therapies," Annals of the Rheumatic Diseases, vol. 73, no. 6, pp. 1138-1143, 2014.

[23] C. L. Krieckaert, A. Jamnitski, M. T. Nurmohamed, P. J. Kostense, M. Boers, and G. Wolbink, "Comparison of long-term clinical outcome with etanercept treatment and adalimumab treatment of rheumatoid arthritis with respect to immunogenicity," Arthritis and Rheumatism, vol. 64, no. 12, pp. 38503855, 2012.

[24] S. B. Krintel, V. P. Grunert, M. L. Hetland et al., "The frequency of anti-infliximab antibodies in patients with rheumatoid arthritis treated in routine care and the associations with adverse drug reactions and treatment failure," Rheumatology, vol. 52, no. 7, pp. 1245-1253, 2013.

[25] D. Pascual-Salcedo, C. Plasencia, S. Ramiro et al., "Influence of immunogenicity on the efficacy of long-term treatment with infliximab in rheumatoid arthritis," Rheumatology, vol. 50, no. 8, pp. 1445-1452, 2011.

[26] T. R. D. J. Radstake, M. Svenson, A. M. Eijsbouts et al., "Formation of antibodies against infliximab and adalimumab strongly correlates with functional drug levels and clinical responses in rheumatoid arthritis," Annals of the Rheumatic Diseases, vol. 68, no. 11, pp. 1739-1745, 2009.

[27] G. J. Wolbink, M. Vis, W. Lems et al., "Development of antiinfliximab antibodies and relationship to clinical response in patients with rheumatoid arthritis," Arthritis \& Rheumatism, vol. 54, no. 3, pp. 711-715, 2006.

[28] F. Atzeni, M. Benucci, S. Sallì, S. Bongiovanni, L. Boccassini, and P. Sarzi-Puttini, "Different effects of biological drugs in rheumatoid arthritis," Autoimmunity Reviews, vol. 12, no. 5, pp. 575-579, 2013.

[29] W. Kievit, J. Fransen, A. J. M. Oerlemans et al., "The efficacy of anti-TNF in rheumatoid arthritis, a comparison between randomised controlled trials and clinical practice," Annals of the Rheumatic Diseases, vol. 66, no. 11, pp. 1473-1478, 2007.

[30] D. Wendling, J.-C. Balblanc, A. Brousse et al., "Surgery in patients receiving anti-tumour necrosis factor $\alpha$ treatment in rheumatoid arthritis: an observational study on 50 surgical procedures," Annals of the Rheumatic Diseases, vol. 64, no. 9, pp. 1378-1379, 2005.

[31] F. Atzeni, P. Sarzi-Puttini, C. Botsios et al., "Long-term antiTNF therapy and the risk of serious infections in a cohort of patients with rheumatoid arthritis: comparison of adalimumab, etanercept and infliximab in the GISEA registry," Autoimmunity Reviews, vol. 12, no. 2, pp. 225-229, 2012.

[32] M. Benucci, G. Saviola, P. Baiardi, M. Manfredi, P. S. Puttini, and F. Atzeni, "Determinants of risk infection during therapy with anti TNF-alpha blocking agents in rheumatoid arthritis," The Open Rheumatology Journal, vol. 6, no. 1, pp. 33-37, 2012.

[33] P. Raaschou, J. F. Simard, M. Holmqvist, and J. Askling, "Rheumatoid arthritis, anti-tumour necrosis factor therapy, and risk of malignant melanoma: nationwide population based prospective cohort study from Sweden," The British Medical Journal, vol. 346, no. 7905, Article ID f1939, 2013.

[34] P. A. van Schouwenburg, T. Rispens, and G. J. Wolbink, "Immunogenicity of anti-TNF biologic therapies for rheumatoid arthritis," Nature Reviews Rheumatology, vol. 9, no. 3, pp. 164-172, 2013.

[35] K. Bendtzen, P. Geborek, M. Svenson, L. Larsson, M. C. Kapetanovic, and T. Saxne, "Individualized monitoring of drug bioavailability and immunogenicity in rheumatoid arthritis patients treated with the tumor necrosis factor $\alpha$ inhibitor infliximab," Arthritis and Rheumatism, vol. 54, no. 12, pp. 37823789, 2006.

[36] A. Jamnitski, G. M. Bartelds, M. T. Nurmohamed et al., "The presence or absence of antibodies to infliximab or adalimumab determines the outcome of switching to etanercept," Annals of the Rheumatic Diseases, vol. 70, no. 2, pp. 284-288, 2011.

[37] F. Atzeni and P. Sarzi-Puttini, "Anti-cytokine antibodies for rheumatic diseases," Current Opinion in Investigational Drugs, vol. 10, no. 11, pp. 1204-1211, 2009.

[38] F. Caprioli, F. Pallone, and G. Monteleone, "Cytokine therapies in Crohn's disease: where are we now and where should we go?" 
Inflammation \& Allergy Drug Targets, vol. 10, no. 1, pp. 47-53, 2011.

[39] H. Radner and D. Aletaha, "Anti-TNF in rheumatoid arthritis: an overview," Wiener Medizinische Wochenschrift, vol. 165, no. 1-2, pp. 3-9, 2015.

[40] G. M. Bartelds, C. L. M. Krieckaert, M. T. Nurmohamed et al., "Development of antidrug antibodies against adalimumab and association with disease activity and treatment failure during long-term follow-up," The Journal of the American Medical Association, vol. 305, no. 14, pp. 1460-1468, 2011.

[41] F. Atzeni, R. Talotta, F. Salaffi et al., "Immunogenicity and autoimmunity during anti-TNF therapy," Autoimmunity Reviews, vol. 12, no. 7, pp. 703-708, 2013.

[42] S. Garcês, J. Demengeot, and E. Benito-Garcia, "The immunogenicity of anti-TNF therapy in immune-mediated inflammatory diseases: a systematic review of the literature with a metaanalysis," Annals of the Rheumatic Diseases, vol. 72, no. 12, pp. 1947-1955, 2013.

[43] K. Bendtzen, M. Ainsworth, C. Steenholdt, O. Ø. Thomsen, and J. Brynskov, "Individual medicine in inflammatory bowel disease: monitoring bioavailability, pharmacokinetics and immunogenicity of anti-tumour necrosis factor-alpha antibodies," Scandinavian Journal of Gastroenterology, vol. 44, no. 7, pp. 774-781, 2009.

[44] M. Chaparro, I. Guerra, P. Muñoz-Linares, and J. P. Gisbert, "Systematic review: antibodies and anti-TNF- $\alpha$ levels in inflammatory bowel disease," Alimentary Pharmacology \& Therapeutics, vol. 35, no. 9, pp. 971-986, 2012.

[45] D.-Y. Chen, Y.-M. Chen, W.-T. Hung et al., "Immunogenicity, drug trough levels and therapeutic response in patients with rheumatoid arthritis or ankylosing spondylitis after 24-week golimumab treatment," Annals of the Rheumatic Diseases, vol. 74, no. 12, pp. 2261-2264, 2015.

[46] P. E. Chandra, J. Sokolove, B. G. Hipp et al., "Novel multiplex technology for diagnostic characterization of rheumatoid arthritis," Arthritis Research and Therapy, vol. 13, no. 3, article R102, 2011.

[47] C. Caruntu, D. Boda, G. Dumitrascu, C. Constantin, and M. Neagu, "Proteomics focusing on immune markers in psoriatic arthritis," Biomarkers in Medicine, vol. 9, no. 6, pp. 513-528, 2015.

[48] M. Svenson, P. Geborek, T. Saxne, and K. Bendtzen, "Monitoring patients treated with anti-TNF- $\alpha$ biopharmaceuticals: assessing serum infliximab and anti-infliximab antibodies," Rheumatology, vol. 46, no. 12, pp. 1828-1834, 2007. 


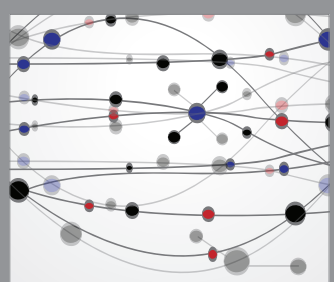

The Scientific World Journal
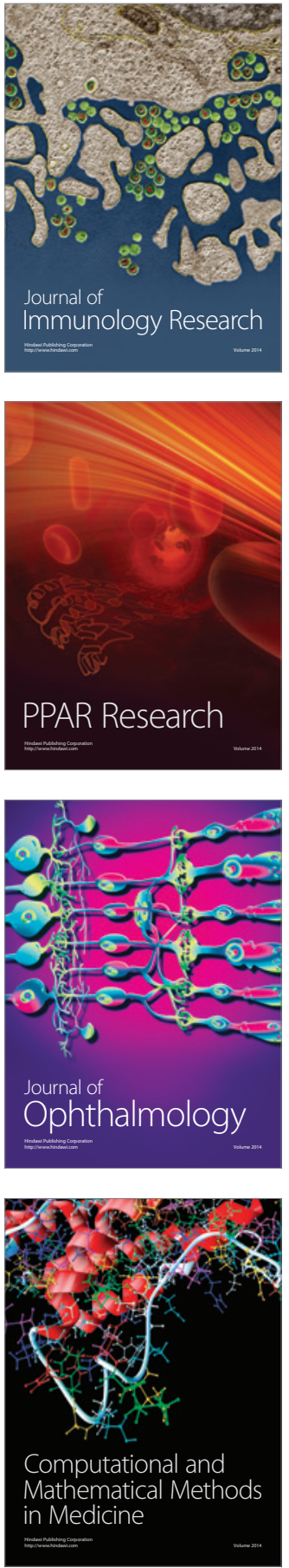

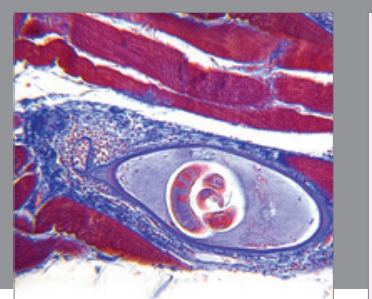

Gastroenterology Research and Practice

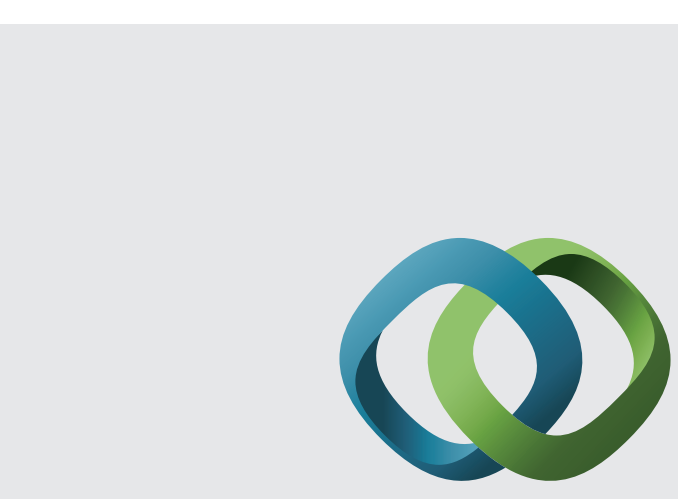

\section{Hindawi}

Submit your manuscripts at

http://www.hindawi.com
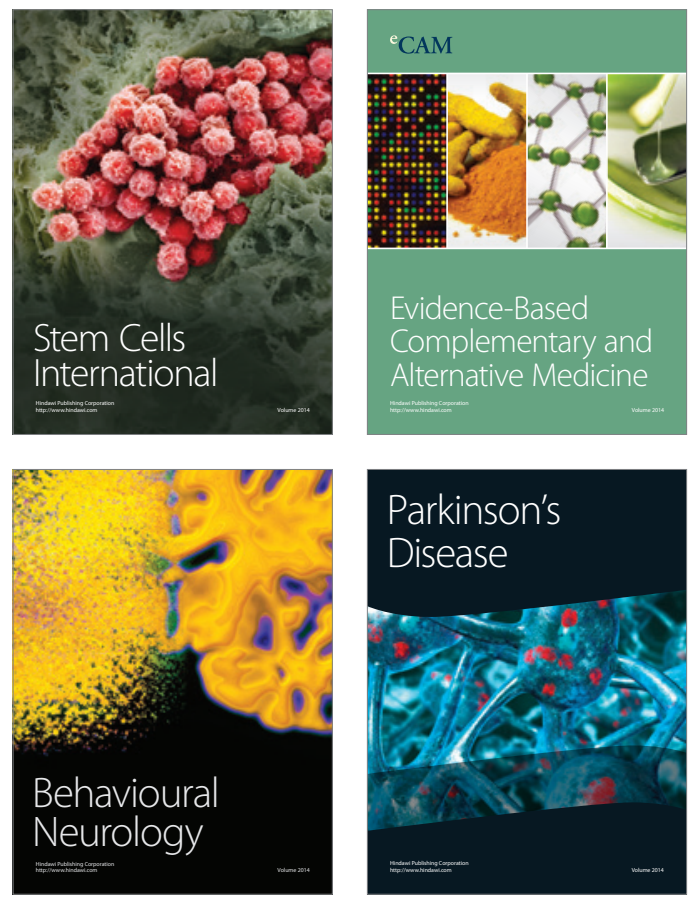
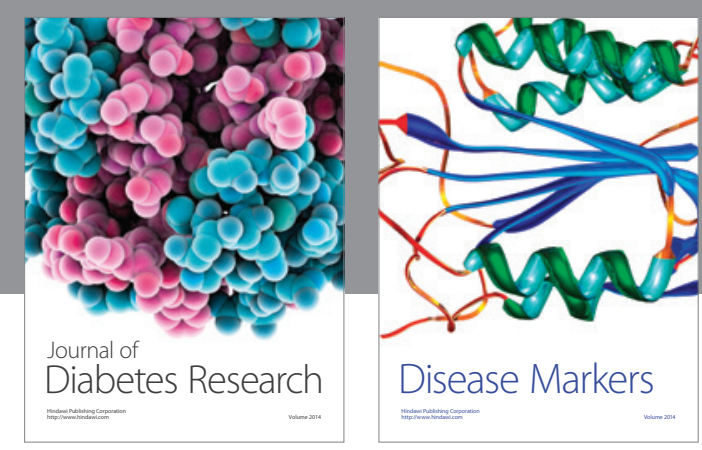

Disease Markers
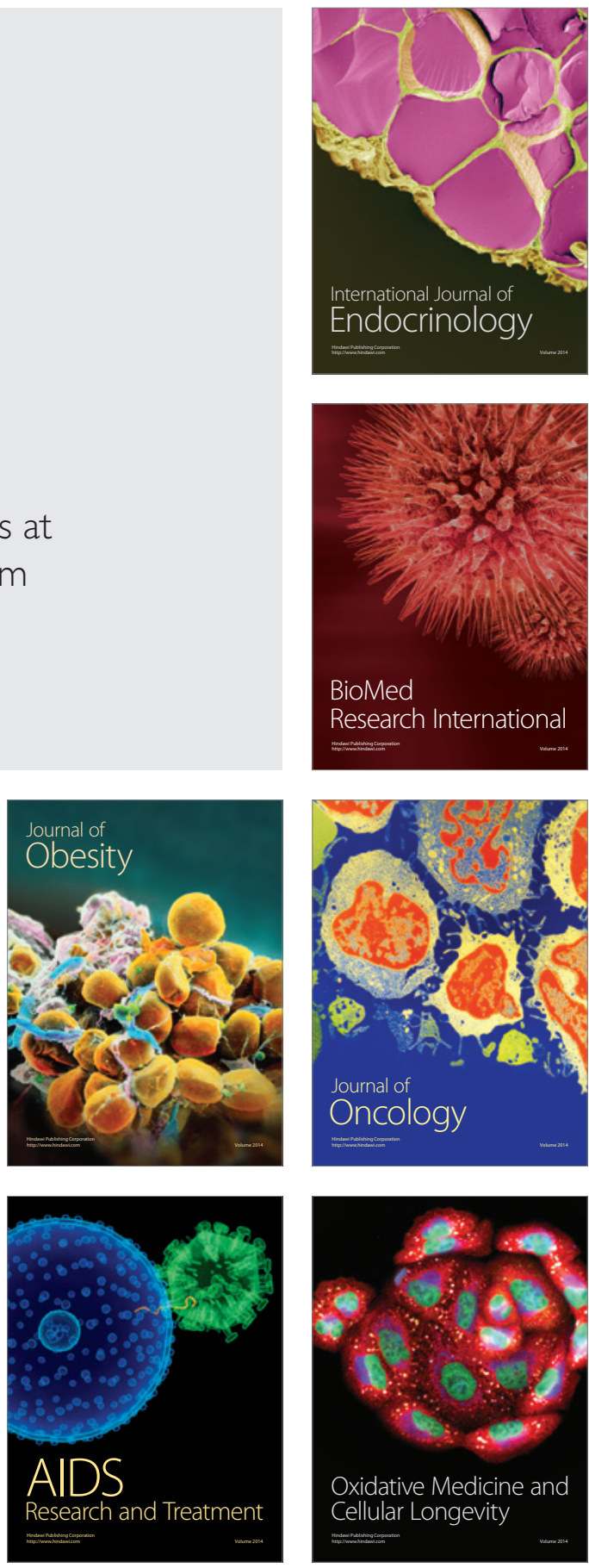